encouraged by OLAW if applicable ${ }^{5}$. If the IACUC finds the use of recently expired buprenorphine does not meet the required outcome or rejects the performance standard concept outright, the only other option would be for the entire project to be placed on hold until the appropriate, in-date buprenorphine drug product is available. $\square$
Oak Ridge, Tennessee, USA.

*e-mail:Joan.Richerson@va.gov

Published online: 23 May 2018

https://doi.org/10.1038/s41684-018-0068-x

References

1. Maguire, J.A., W. Jiang, and L.X. Yu. 2017. Chapter 40- Modern pharmaceutical regulations: quality assessment for drug products. Published in Developing Solid Oral Dosage Forms (2nd Edition). Editors: Y. Qiu, Y. Chen, G.G.Z. Zhang, L. Yu, and R.V. Mantri. Academic Press, Tokyo, pp. 1095-1126. https://www.sciencedirect. $\mathrm{com} /$ topics/medicine-and-dentistry/drug-reference-standard
2. United States Pharmacopeial Convention. Position statement found on-line at: http://www.usp.org/sites/ default/files/usp/document/our-work/reference-standards/ qa-policy-statement-usp-rs-uses-and-applications.pdf (2017).

3. Office of Laboratory Animal Welfare, National Institutes of Health. Frequently asked questions: PHS Policy on humane care and use of laboratory animals. F.5. https://grants.nih.gov/grants/ OLAW/faqs.htm\#663 (2017).

4. Institute for Laboratory Animal Research. Guide for the Care and Use of Laboratory Animals. 8th edn., pp. 6-7 (National Academies Press, Washington, DC, 2011).

5. Office of Laboratory Animal Welfare, National Institutes of Health. Frequently asked questions: PHS Policy on humane care and use of laboratory animals. G.10. https://grants.nih.gov/grants/ olaw/faqs.htm\#3284 (2017).

\title{
Considering animal welfare with scientific justification in mind
}

\footnotetext{
T
} he scenario has two components to be considered: animal welfare and scientific justification.

In consideration of animal welfare, the IACUC should not agree with the use of the outdated drug or the reference standard. The US Pharmacopeia clearly states that the reference standards are meant to be used in tests and assays, and not in humans and animals ${ }^{1}$. Therefore, the safety and the pain control properties of the reference standard are not guaranteed in rodents; using it in this study could risk unexpected adverse effects or insufficient pain control. Outdated buprenorphine $\mathrm{HCl}$ should also not be used; while there may be literature that vouches for acceptable efficacy in outdated buprenorphine $\mathrm{HCl}$, the reality could depend on many variables, such as the manufacturer, the length of expiration, and the specific species, sex, and strains of the animals. Furthermore, approving the usage of USP or outdated drugs comes with two risks: first, this may set a precedent for future similar requests; second, if the rodents that received USP or outdated buprenorphine $\mathrm{HCl}$ suffered an adverse event, the IACUC and the veterinarian could be held responsible. According to the Guide for the Care and Use of Laboratory Animals, expired anesthetic and analgesic agents should not be used ${ }^{2}$. Taken together, the IACUC should not approve this request.

Another reason why the request from the Rosenfeld lab should not be approved is inadequate scientific justification. Using either of these choices would create a variable that could render the results incomparable with previous work. If Dr. Rosenfeld does not wish to use a different negative control in the middle of the study, then neither USP nor outdated buprenorphine $\mathrm{HCl}$ should be used. Creating variables like this could result in unusable data and unnecessary use of animals. The IACUC could instead suggest the lab use buprenorphine SR (assuming that only the $\mathrm{HCl}$ formulation is in shortage), if this formulation is acceptable for the lab.

The use of VVC is initially correct in this case because the consultation was for using a different drug in response to a shortage of the original one, which falls within the parameter of a $\mathrm{VVC}^{3}$. However, because the lab's request to use USP or outdated buprenorphine $\mathrm{HCl}$ is not based on the need for pain management, this issue becomes one where the PI needs to scientifically justify the use of alternative drugs at the risk of animal welfare. Therefore, the veterinarian is correct in bringing this issue to the IACUC.

In the event of drug shortage of unknown duration, it is understandable that investigators would want to carry on the project instead of waiting indefinitely. The IACUC must not forget that animal lives could be wasted too if the project is delayed. Prior to making a definitive decision, the IACUC must carefully consider the justification provided by the investigators. $\square$

Yvonne R. Cornejo, Leo Holguin and Jui Tu* Center for Comparative Medicine, City of Hopel Beckman Research Institute, Duarte,

California, USA.

*e-mail: jutu@coh.org

Published online: 23 May 2018

https://doi.org/10.1038/s41684-018-0070-3

References

1. United States Pharmacopeial Convention. FAQS: Reference Standards. http://www.usp.org/frequently-asked-questions/ reference-standards

2. Institute for Laboratory Animal Research. Guide for the Care and Use of Laboratory Animals 8th edn. (National Academies Press, Washington, DC, 2011).

3. National Institutes of Health. Guidance on Significant Changes to Animal Activities. Notice NOT-OD-14-126. (National Institutes of Health, Washington, DC, 26 August 2014).

\section{Redirecting the request to the IACUC}

D ue to the shortage of pharmaceuticalgrade buprenorphine, the IACUC and Attending Veterinarian are faced with the challenge of determining which situation is more appropriate: allow Dr. Rosenfeld to use non-pharmaceutical-grade buprenorphine, allow her to use expired pharmaceutical-grade buprenorphine, or allow neither, postponing the research until pharmaceutical-grade buprenorphine becomes available.
Guidance provided by OLAW indicates that use of expired analgesics is inconsistent with acceptable veterinary practices and adequate veterinary care. OLAW does not provide for the use of analgesics beyond their expiration date under any 